Homology, Homotopy and Applications, vol.10(1), 2008, pp.345-351

\title{
STRONG EXCISION AND STRONG SHAPE INVARIANCE ARE EQUIVALENT FOR HOMOLOGY THEORIES ON THE CATEGORY OF COMPACT METRIC PAIRS
}

\author{
PETER MROZIK
}

(communicated by Brooke Shipley)

\begin{abstract}
In 1960, Milnor gave an axiomatic characterization of Steenrod homology as an ordinary homology theory on the category of compact metric pairs satisfying the strong excision axiom and the cluster axiom. The subject of this paper is to explore the role of the strong excision axiom on its own. It is proved that for any homology theory on the category of compact metric pairs strong excision is equivalent to strong shape invariance.
\end{abstract}

\section{Introduction}

Let $\boldsymbol{C} \boldsymbol{M}^{2}$ be the category of compact metric pairs and continuous maps of pairs. The category $\boldsymbol{C M}$ of compact metric spaces and continuous maps will be regarded as a full subcategory of $\boldsymbol{C} \boldsymbol{M}^{2}$ by identifying the pair $(X, \emptyset)$ with the space $X$. Let $R: \boldsymbol{C M}^{\mathbf{2}} \rightarrow \boldsymbol{C} \boldsymbol{M}$ be the restriction functor $(R(X, A)=A)$, and let $\boldsymbol{A b}$ be the category of abelian groups. A homology theory on $\boldsymbol{C} \boldsymbol{M}^{\mathbf{2}}$ is a collection of covariant functors $H_{n}: \boldsymbol{C M}^{\mathbf{2}} \rightarrow \boldsymbol{A} \boldsymbol{b}$ and natural transformations $\partial: H_{n+1} \rightarrow H_{n} \circ R, n \in \mathbb{Z}$, satisfying the axioms of homotopy invariance, exactness and excision.

In 1960 Milnor [9] showed that (ordinary) Steenrod homology can be characterized uniquely as a homology theory satisfying the dimension axiom, the strong excision axiom (SE) and the cluster axiom $(C)$ :

(SE) For each compact metric pair $(X, A)$ with $A \neq \emptyset$, the quotient map

$$
p:(X, A) \rightarrow(X / A, *)
$$

induces isomorphisms $p_{*}: H_{n}(X, A) \rightarrow H_{n}(X / A, *)$ for all $n \in \mathbb{Z}$.

(C) For each sequence $\left(X_{i}, *\right), i \in \mathbb{N}$, of pointed compact metric spaces, the projections $p_{k}: C \boldsymbol{l}_{i=1}^{\infty}\left(X_{i}, *\right) \rightarrow\left(X_{k}, *\right)$ defined on the cluster $\boldsymbol{C l}_{i=1}^{\infty}\left(X_{i}, *\right)$ induce isomorphisms

$$
\pi_{n}: H_{n}\left(\boldsymbol{C l}_{i=1}^{\infty}\left(X_{i}, *\right)\right) \rightarrow \prod_{i=1}^{\infty} H_{n}\left(X_{i}, *\right)
$$

for all $n \in \mathbb{Z}$.

Received February 23, 2008, revised April 6, 2008; published on May 12, 2008.

2000 Mathematics Subject Classification: 55P55, 55N20, 55N40.

Key words and phrases: strong shape theory, homology theory, strong excision axiom.

This article is available at http://intlpress.com/HHA/v10/n1/a15

Copyright (c) 2008, International Press. Permission to copy for private use granted. 
Remark 1.1. The concept of strong excision appeared first in 1952 in [12]. In [12] Wallace proved that Alexander cohomology has the so-called map excision property. This property is now better known under the name relative homeomorphism axiom $(R H)$. In its homological version on $\boldsymbol{C} \boldsymbol{M}^{\mathbf{2}}$ it says the following:

(RH) Each relative homeomorphism $h:(X, A) \rightarrow(Y, B)$ between compact metric pairs induces isomorphisms $h_{*}: H_{n}(X, A) \rightarrow H_{n}(Y, B)$ for all $n \in \mathbb{Z}$.

Recall that a map of pairs $h:(X, A) \rightarrow(Y, B)$ is a relative homeomorphism if $h$ maps $X \backslash A$ homeomorphically onto $Y \backslash B$. On $\boldsymbol{C} \boldsymbol{M}^{\mathbf{2}},(\mathrm{RH})$ and (SE) are obviously equivalent. In Milnor's original paper [9], $(\mathrm{RH})$ appears instead of (SE).

In the almost 50 years since Milnor's axiomatic characterization of Steenrod homology a lot of work has been done to generalize his result. See e.g. [2, 3, 5], especially Bauer's papers $[\mathbf{2}, \mathbf{3}]$, which led to a deeper understanding of so-called generalized Steenrod homology theories and their relation to strong shape theory. In particular, Bauer showed that each homology theory, which is represented by a CW-spectrum with certain mild connectivity properties and which satisfies the strong excision axiom and the cluster axiom, extends over the strong shape category of compact metric pairs. For instance, this applies to all generalized Steenrod homology theories.

Thus, roughly speaking, the strong excision axiom and the cluster axiom together imply strong shape invariance. However, this leaves the question which property it is precisely that forces a homology theory to be strong shape invariant. One might be tempted to think that the cluster axiom, which is a weak continuity property allowing a simple form of approximation of spaces, could be the reason. But it turns out, perhaps surprisingly, that it is the strong excision axiom on its own which is equivalent to strong shape invariance. See Theorem 2.3 below.

\section{The strong excision axiom and strong shape invariance}

We assume that the reader is familiar with strong shape theory (see e.g. $[\mathbf{1}, \mathbf{7}]$ ). Let $\boldsymbol{S} \boldsymbol{S h}^{\mathbf{2}}$ be the strong shape category of compact metric pairs and $S: \boldsymbol{C M}^{\mathbf{2}} \rightarrow \boldsymbol{S} \boldsymbol{S h}^{\mathbf{2}}$ be the strong shape functor. The category $\boldsymbol{S} \boldsymbol{S h}^{\mathbf{2}}$ is perhaps not as well-known as the strong shape category $\boldsymbol{S S h}$ of compact metric spaces. Fortunately, all we have to know about it here are the following two facts:

Fact 1. The functor $S: C \boldsymbol{M}^{2} \rightarrow \boldsymbol{S} \boldsymbol{S h}^{2}$ localizes $\boldsymbol{C} \boldsymbol{M}^{2}$ at the class of strong shape equivalences of pairs of compact metric spaces.

Fact 2. A map of pairs $f:(X, A) \rightarrow(Y, B)$ is a strong shape equivalence of pairs if and only if the absolute map $f: X \rightarrow Y$ and the restriction $R(f): A \rightarrow B$ are strong shape equivalences of compact metric spaces.

Concerning Fact 1 see the Appendix. For Fact 2 see [6] (cf. also [7, Theorem 10]).

Definition 2.1. A homology theory $\left(H_{n}, \partial\right)$ on $\boldsymbol{C} \boldsymbol{M}^{\mathbf{2}}$ is called strong shape invariant if it satisfies the following axiom:

(SSI) For each strong shape equivalence of pairs $f:(X, A) \rightarrow(Y, B)$, the induced morphisms $f_{*}: H_{n}(X, A) \rightarrow H_{n}(Y, B)$ are isomorphisms for all $n \in \mathbb{Z}$.

Remark 2.2. As a consequence of Fact 1, (SSI) is equivalent to the existence of covariant functors $h_{n}: \boldsymbol{S} \boldsymbol{S h}^{\mathbf{2}} \rightarrow \boldsymbol{A} \boldsymbol{b}$ such that $H_{n}=h_{n} \circ S$ for all $n \in \mathbb{Z}$. These functors $h_{n}$ are of course uniquely determined. 
Theorem 2.3. A homology theory $\left(H_{n}, \partial\right)$ on $\boldsymbol{C M}^{\mathbf{2}}$ is strong shape invariant if and only if it satisfies the strong excision axiom.

Proof. 1) Let $\left(H_{n}, \partial\right)$ satisfy the strong excision axiom. It is known from [10] that the strong shape functor $S: \boldsymbol{C M} \rightarrow \boldsymbol{S} \boldsymbol{S}$ localizes $\boldsymbol{C M}$ at the class of cylinder base embeddings. Recall from [10] that a closed subspace $B$ of a compact metric space $C$ is called a cylinder base if $C \backslash B \approx X \times(0,1]$ for some compact metric space $X$. Consider such a cylinder base $B \subset C$. By strong excision we have isomorphisms $p_{*}: H_{n}(C, B) \rightarrow H_{n}(C / B, *)$ for all $n \in \mathbb{Z}$. But $C / B$ is nothing but the cone $C X$ on $X$. This implies $H_{n}(C / B, *)=H_{n}(C X, *)=0$. Hence $H_{n}(C, B)=0$. The long exact sequence of the pair $(C, B)$

$$
\rightarrow H_{n+1}(C, B) \rightarrow H_{n}(B) \rightarrow H_{n}(C) \rightarrow H_{n}(C, B) \rightarrow
$$

shows then that inclusion induces isomorphisms $H_{n}(B) \rightarrow H_{n}(C)$ in all dimensions. That is, the absolute functors $H_{n}: \boldsymbol{C} \boldsymbol{M} \rightarrow \boldsymbol{A} \boldsymbol{b}$ take cylinder base embeddings to isomorphisms. Therefore the absolute functors $H_{n}$ expand uniquely to functors $h_{n}: \boldsymbol{S} \boldsymbol{S} \boldsymbol{h} \rightarrow \boldsymbol{A b}$ such that $H_{n}=h_{n} \circ S$ for all $n \in \mathbb{Z}$. A fortiori the absolute functors $H_{n}$ take all strong shape equivalences to isomorphisms. Next consider a strong shape equivalence of pairs $f:(X, A) \rightarrow(Y, B)$. By Fact 2, the absolute map $f: X \rightarrow Y$ and the restriction $R(f): A \rightarrow B$ are strong shape equivalences of compact metric spaces. The long exact sequences of the pairs $(X, A)$ and $(Y, B)$ and the induced morphisms $f_{*}: H_{n}(X, A) \rightarrow H_{n}(Y, B), f_{*}: H_{n}(X) \rightarrow H_{n}(Y)$, and $R(f)_{*}: H_{n}(A) \rightarrow$ $H_{n}(B)$ form an infinite commutative diagram. Now, $f_{*}: H_{n}(X) \rightarrow H_{n}(Y)$ and $R(f)_{*}: H_{n}(A) \rightarrow H_{n}(B)$ are isomorphisms. The Five Lemma shows then that all $f_{*}: H_{n}(X, A) \rightarrow H_{n}(Y, B)$ are isomorphisms. This means that $\left(H_{n}, \partial\right)$ is strong shape invariant.

2) Let $\left(H_{n}, \partial\right)$ be strong shape invariant. Consider a pair $(X, A)$ with $A \neq \emptyset$. Let $C A$ be the cone on $A$, where $A$ is identified with the base of $C A$. It is well-known that the inclusion $i:(X, A) \rightarrow(X \cup C A, C A)$ induces isomorphisms in homology (this is a consequence of ordinary excision). The quotient map

$$
q:(X \cup C A, C A) \rightarrow((X \cup C A) / C A, *)=(X / A, *)
$$

is a strong shape equivalence of pairs (recall Fact 2 and apply [7, Corollary 10.8]). This implies that the quotient map $p:(X, A) \rightarrow(X / A, *)$ induces isomorphisms in homology since $p=q \circ i$. This means that $\left(H_{n}, \partial\right)$ satisfies the strong excision axiom.

Remark 2.4. Of course, Theorem 2.3 has a version for cohomology. Details can safely be left to the reader.

As a final application we observe that the homology theory $\epsilon_{*}$ which has been constructed by Brown, Douglas and Fillmore [4] satisfies the strong excision axiom.

Corollary 2.5. The homology theory $\epsilon_{*}$ is strong shape invariant.

Remark 2.6. Bauer has shown that $\epsilon_{*}$ is naturally isomorphic to strong shape $\overline{B U}_{*^{-}}$ homology on the category of finite-dimensional compact metric spaces. Whether this is true for all compact metric spaces remains open. 


\section{Appendix A. Representing $S S h^{2}$ as a quotient category}

It is well-known that in the absolute case the strong shape functor $S: \boldsymbol{M} \rightarrow \boldsymbol{S S h}$ localizes $\boldsymbol{C M}$ at the class of strong shape equivalences (see e.g. $[\mathbf{7}, \S 10.7]$ ). This is also true for pairs (Fact 1), but it seems that there is no reference in the literature. The Appendix is therefore devoted to supply a proof of Fact 1. We only give a sketch since for pairs everything works completely analogous to the absolute case where all the necessary steps have been published in detail.

There are various different constructions of the strong shape category $\boldsymbol{S} \boldsymbol{S h}$ (yielding isomorphic categories when restricted to compact metric spaces). All these constructions have analogues for pairs, but not all of them have been presented in the literature. As the standard construction of $\boldsymbol{S} \boldsymbol{S h}^{\mathbf{2}}$ we can regard that of $[\mathbf{7}, \S 8.2]$ (see also [8]). The morphisms $(X, A) \rightarrow(Y, B)$ in $\boldsymbol{S} \boldsymbol{S h}^{\mathbf{2}}$ are determined by coherent homotopy classes of coherent maps between cofinite strong $\mathbf{H P o l}_{\mathbf{2}}$-expansions of $(X, A)$ and $(Y, B)$ (cf. [7, Remark 7.20]). For compact metric pairs one can choose these $\mathbf{H P o l}_{2}$-expansions to be inverse sequences of compact polyhedral pairs whose inverse limit is $(X, A) \operatorname{resp} .(Y, B)$.

For our purposes it will be convenient to work with the Quigley strong shape category of compact metric pairs which we shall denote by $\boldsymbol{Q} \boldsymbol{S} \boldsymbol{S h}^{\mathbf{2}}$ (see [11] for the absolute case). The objects of $\boldsymbol{Q} \boldsymbol{S} \boldsymbol{S h}^{2}$ are all compact pairs $(X, A)$ such that $X \subset Q$, where $Q$ is the Hilbert cube. To define morphisms, we proceed as follows.

A map $\varphi: Q \times(0,1] \rightarrow Q$ is called an approaching map from $(Q, X, A)$ to $(Q, Y, B)$, written as $\varphi:(Q, X, A) \Rightarrow(Q, Y, B)$, if each pair of neighbourhoods $\left(V, V^{\prime}\right)$ of $(Y, B)$ in $Q$ admits a pair of neighbourhoods $\left(U, U^{\prime}\right)$ of $(X, A)$ in $Q$ and an $\epsilon>0$ such that $\varphi\left(\left(U, U^{\prime}\right) \times(0, \epsilon]\right) \subset\left(V, V^{\prime}\right)$. An approaching homotopy between approaching maps is defined in the obvious way as an approaching map

$$
\phi:(Q, X, A) \times I=(Q \times I, X \times I, A \times I) \Rightarrow(Q, Y, B),
$$

i.e. a map $\phi: Q \times I \times(0,1] \rightarrow Q$ such that each pair of neighbourhoods $\left(V, V^{\prime}\right)$ of $(Y, B)$ in $Q$ admits a pair of neighbourhoods $\left(U, U^{\prime}\right)$ of $(X, A)$ in $Q$ and an $\epsilon>0$ such that $\phi\left(\left(U, U^{\prime}\right) \times I \times(0, \epsilon]\right) \subset\left(V, V^{\prime}\right)$; cf. $[\mathbf{7}, \mathbf{1 0}, \mathbf{1 1}]$.

The set of morphisms $\boldsymbol{Q} \boldsymbol{S} \boldsymbol{S h}^{2}((X, A),(Y, B))$ is then defined as the set of approaching homotopy classes of approaching maps $\varphi:(Q, X, A) \Rightarrow(Q, Y, B)$.

The resulting category $\boldsymbol{Q} \boldsymbol{S} \boldsymbol{S h}^{2}$ is equivalent to the strong shape category $\boldsymbol{S S h}^{2}$ as described above. This can be shown by copying the arguments of [7, §9.1]. Under this equivalence the strong shape functor $S: C \boldsymbol{M}^{\mathbf{2}} \rightarrow \boldsymbol{S} \boldsymbol{S h}^{\mathbf{2}}$ corresponds to the Quigley strong shape functor $Q S: C \boldsymbol{M}^{2}(Q) \rightarrow Q S S h^{2}$, where $C \boldsymbol{M}^{2}(\boldsymbol{Q})$ is the full subcategory of $\boldsymbol{C} \boldsymbol{M}^{\mathbf{2}}$ having the same objects as $\boldsymbol{Q S S \boldsymbol { h } ^ { 2 }}$. To see what $Q S(f)$ is for a given map $f:(X, A) \rightarrow(Y, B)$, choose any extension $F: Q \rightarrow Q$ of $f: X \rightarrow Y$. Define $\varphi: Q \times(0,1] \rightarrow Q$ by $\varphi(x, t)=F(x)$. This is an approaching map $\varphi:(Q, X, A) \Rightarrow$ $(Q, Y, B)$, and we have $Q S(f)=[\varphi]$, where [.] denotes approaching homotopy class. For later use, let us call $\varphi$ an approaching extension of $f$.

By a cylinder base embedding of pairs we mean an embedding of compact metric pairs $f:\left(B, B_{0}\right) \rightarrow\left(C, C_{0}\right)$ such that

$$
\left(C, C_{0}\right) \backslash f\left(B, B_{0}\right)=\left(C \backslash f(B), C_{0} \backslash f\left(B_{0}\right)\right) \approx\left(X, X_{0}\right) \times(0,1]
$$


for some compact metric pair $\left(X, X_{0}\right)$. This concept has been introduced in [10] for the absolute case. Copying the proof of [10, Theorem 4.2], we obtain

Theorem A.1. Each cylinder base embedding $f:\left(B, B_{0}\right) \rightarrow\left(C, C_{0}\right)$ in $\boldsymbol{C M}^{\mathbf{2}}(\boldsymbol{Q})$ is a strong shape equivalence of pairs, i.e. $Q S(f)$ is an isomorphism in $\boldsymbol{Q S S \boldsymbol { h } ^ { 2 }}$.

The concept of cylinder base embeddings generalizes the concept of mapping cylinders of approaching maps $\varphi:(Q, X, A) \Rightarrow(Q, Y, B)$ (cf. [10]). For an approaching map of pairs, the mapping cylinder $C^{2}(\varphi)$ is the pair $\left(C\left(\varphi_{\text {abs }}\right),\left(C\left(\varphi_{\text {rel }}\right)\right)\right.$, where $\varphi_{\text {abs }}:(Q, X) \Rightarrow(Q, Y)$ is $\varphi$ considered as an ordinary approaching map $(Q, X) \Rightarrow$ $(Q, Y)$ and $\varphi_{\text {rel }}:(Q, A) \Rightarrow(Q, B)$ is $\varphi$ considered as an ordinary approaching map $(Q, A) \Rightarrow(Q, B)$ and $C\left(\varphi_{a b s}\right), C\left(\varphi_{\text {rel }}\right)$ are the corresponding mapping cylinders as constructed in [10]. As sets we have $C^{2}(\varphi)=(X, A) \times(0,1]+(Y, B)$, where + denotes disjoint union. The right-hand side is given a compact metric topology depending on $\varphi$ such that $(X, A) \times(0,1]$ and $(Y, B)$ retain their original topologies. We then have two canonical embeddings $i_{(X, A)}:(X, A) \rightarrow C^{2}(\varphi)$ and $j_{(Y, B)}:(Y, B) \rightarrow$ $C^{2}(\varphi)\left(i_{(X, A)}\right.$ identifies $(X, A)$ with the top $(X, A) \times\{1\}$ of $\left.C^{2}(\varphi)\right)$. Since $C^{2}(\varphi)$ is a pair of compact metric spaces, we may assume that $C^{2}(\varphi)$ is an object of $\boldsymbol{Q} \boldsymbol{S} \boldsymbol{S h}^{2}$. By the construction of the mapping cylinder, we have the following result.

Theorem A.2. $j_{(Y, B)}$ is a cylinder base embedding of pairs. $[\varphi]$ decomposes as

$$
[\varphi]=Q S\left(j_{(Y, B)}\right)^{-1} \circ Q S\left(i_{(X, A)}\right)
$$

The proof can be copied from that of [10, Corollary 6.4].

Remark $A$.3. Let $f:(X, A) \rightarrow(Y, B)$ be a map of pairs and $\varphi:(Q, X, A) \Rightarrow(Q, Y, B)$ be an arbitrary approaching extension of $f$. Then $C^{2}(\varphi)$ is the ordinary mapping cylinder $C^{2}(f)=(C(f), C(R(f))$ of $f$ (cf. Proposition 2.5 of $[\mathbf{1 0}])$.

In [10] we introduced the concept of a generalized calculus of left fractions. We do not repeat the definition here but recommend the reader to consult [10]. Let $\boldsymbol{H} \boldsymbol{C} \boldsymbol{M}^{2}(\boldsymbol{Q})$ be the homotopy category of $\boldsymbol{C M}^{\mathbf{2}}(\boldsymbol{Q})$ (the morphisms are homotopy classes of maps of pairs $f:(X, A) \rightarrow(Y, B)$, where of course all homotopies are required to be maps of pairs $H:(X, A) \times I \rightarrow(Y, B))$. Let $H: \boldsymbol{C M}^{\mathbf{2}}(\boldsymbol{Q}) \rightarrow$ $\boldsymbol{H} \boldsymbol{C} \boldsymbol{M}^{2}(\boldsymbol{Q})$ be the canonical functor taking maps to homotopy classes. Let $\Sigma_{C B E}$ be the set of cylinder base embeddings of compact metric pairs contained in the Hilbert cube $Q$ and let $H \Sigma_{C B E}$ be the set of homotopy classes of maps in $\Sigma_{C B E}$. Theorem 6.5 of $[\mathbf{1 0}]$ easily generalizes to

Theorem A.4. $H \Sigma_{C B E}$ admits a generalized calculus of left fractions in $\boldsymbol{H C M}^{\mathbf{2}}(\boldsymbol{Q})$.

Fact 1 is covered by

Theorem A.5. $Q S: C M^{2}(Q) \rightarrow Q S S h^{2}$ localizes $C M^{2}(Q)$ at $\Sigma_{C B E}$. A fortiori $Q S$ localizes $C \boldsymbol{M}^{2}(\boldsymbol{Q})$ at strong shape equivalences of compact metric pairs in $Q$.

Proof. Let us first consider any functor $\Theta: C M^{2}(Q) \rightarrow \boldsymbol{K}$ taking $\Sigma_{C B E}$ to isomorphisms in the category $\boldsymbol{K}$. Then $\Theta$ is homotopy invariant, i.e. factorizes as $\Theta=H \Theta \circ H$ with $H \Theta: \boldsymbol{H}_{\boldsymbol{C}} \boldsymbol{M}^{\mathbf{2}}(\boldsymbol{Q}) \rightarrow \boldsymbol{K}$. This comes from the fact that the inclusions $i_{k}:(X, A) \rightarrow(X, A) \times I, i_{k}(x)=(x, k), k=0,1$ are cylinder base embeddings 
of pairs and therefore induce isomorphims $\Theta\left(i_{k}\right)$. Hence $\Theta\left(i_{0}\right)=\Theta\left(i_{1}\right)$ since $\Theta(p)$, where $p:(X, A) \times I \rightarrow(X, A)$ denotes the projection map, is a common right inverse for both. This implies that homotopic maps have the same image under $\Theta$.

It is therefore sufficient to show that the functor $H Q S: \boldsymbol{H C M}^{2}(\boldsymbol{Q}) \rightarrow \boldsymbol{Q S S \boldsymbol { S } ^ { 2 }}$ localizes $\boldsymbol{H} \boldsymbol{C} \boldsymbol{M}^{2}(\boldsymbol{Q})$ at $H \Sigma_{C B E}$.

Let $\boldsymbol{H} \boldsymbol{C} \boldsymbol{M}^{2}(\boldsymbol{Q}) / H \Sigma_{C B E}$ be the standard category of left fractions and let $\Lambda: \boldsymbol{H C M}^{2}(\boldsymbol{Q}) \rightarrow \boldsymbol{H C M}^{2}(\boldsymbol{Q}) / H \Sigma_{C B E}$ be the quotient functor which localizes $\boldsymbol{H} \boldsymbol{C} \boldsymbol{M}^{2}(\boldsymbol{Q})$ at $H \Sigma_{C B E}$. Because $H Q S$ takes $H \Sigma_{C B E}$ to isomorphims (Theorem 3.1), there exists a unique functor $\Omega: \boldsymbol{H C M}^{2}(\boldsymbol{Q}) / H \Sigma_{C B E} \rightarrow \boldsymbol{Q S} \boldsymbol{S h}^{2}$ such that $H Q S=$ $\Omega \circ \Lambda$.

By Theorem 3.3, $\Omega$ is a full functor. To show that $\Omega$ is faithful, consider two left fractions $\left[e_{k}\right] \backslash\left[f_{k}\right] \in \boldsymbol{H} \boldsymbol{C} \boldsymbol{M}^{\mathbf{2}}(\boldsymbol{Q}) / H \Sigma_{C B E}((X, A),(Y, B))$ such that $\Omega\left(\left[e_{0}\right] \backslash\left[f_{0}\right]\right)=$ $\Omega\left(\left[e_{1}\right] \backslash\left[f_{1}\right]\right)$. Here, $\left[f_{k}\right]:(X, A) \rightarrow\left(Z_{k}, C_{k}\right)$ and $\left[e_{k}\right]:(Y, B) \rightarrow\left(Z_{k}, C_{k}\right)$ are homotopy classes such that $\left[e_{k}\right] \in H \Sigma_{C B E}$. Since $H \Sigma_{C B E}$ admits a generalized calculus of left fractions there are $\left[v_{k}\right]:\left(Z_{k}, C_{k}\right) \rightarrow\left(Z^{\prime}, C^{\prime}\right)$ such that $\left[v_{0}\right] \circ\left[e_{0}\right]=\left[v_{1}\right] \circ\left[e_{1}\right]$ and $\left[v_{0}\right] \in H \Sigma_{C B E}$. Moreover, there exists $[w]:\left(Z^{\prime}, C^{\prime}\right) \rightarrow(Z, C)$ such that $[w] \circ\left[v_{0}\right] \circ\left[e_{0}\right] \in H \Sigma_{C B E}$ (see $[\mathbf{1 0}]$ for the latter property). Let $[e]=[w] \circ\left[v_{0}\right] \circ\left[e_{0}\right]$ and $\left[g_{k}\right]=[w] \circ\left[v_{k}\right] \circ\left[f_{k}\right]$. By construction, $\left[e_{k}\right] \backslash\left[f_{k}\right]=[e] \backslash\left[g_{k}\right]$. Thus

$$
H Q S([e])^{-1} \circ H Q S\left(\left[g_{0}\right]\right)=\Omega\left([e] \backslash\left[g_{0}\right]\right)=\Omega\left([e] \backslash\left[g_{1}\right]\right)=H Q S([e])^{-1} \circ H Q S\left(\left[g_{1}\right]\right),
$$

whence $H Q S\left(\left[g_{0}\right]\right)=H Q S\left(\left[g_{1}\right]\right)$. We now construct $\left[e^{\prime}\right] \in H \Sigma_{C B E}$ such that $\left[e^{\prime}\right] \circ\left[g_{0}\right]=\left[e^{\prime}\right] \circ\left[g_{1}\right]$. This will prove that $\Omega$ is faithful since we can choose $\left[w^{\prime}\right]$ such that $\left[w^{\prime}\right] \circ\left[e^{\prime}\right] \circ[e] \in H \Sigma_{C B E}$ which implies that

$$
\begin{aligned}
{[e] \backslash\left[g_{0}\right] } & =\left(\left[w^{\prime}\right] \circ\left[e^{\prime}\right] \circ[e]\right) \backslash\left(\left[w^{\prime}\right] \circ\left[e^{\prime}\right] \circ\left[g_{0}\right]\right) \\
& \left.=\left[w^{\prime}\right] \circ\left[e^{\prime}\right] \circ[e]\right) \backslash\left(\left[w^{\prime}\right] \circ\left[e^{\prime}\right] \circ\left[g_{1}\right]\right)=[e] \backslash\left[g_{1}\right] .
\end{aligned}
$$

To get $\left[e^{\prime}\right]$, choose approaching extensions $\varphi_{k}:(Q, X, A) \Rightarrow(Q, Z, C)$ of $g_{k}:(X, A) \rightarrow$ $(Z, C)$. By assumption, there exists an approaching homotopy

$$
\phi:(Q, X, A) \times I \Rightarrow(Q, Z, C)
$$

connecting $\varphi_{0}$ and $\varphi_{1}$. We identify the mapping cylinders $C^{2}\left(\varphi_{k}\right)=C^{2}\left(g_{k}\right)$ with the left and right face of the mapping cylinder $C^{2}(\phi)$ (as sets, $C^{2}\left(g_{k}\right)=(X, A) \times\{k\}+$ $\left.(Z, C) \subset(X, A) \times I+(Z, C)=C^{2}(\phi)\right)$. Let $\alpha_{k}: C^{2}\left(g_{k}\right) \rightarrow C^{2}(\phi)$ be the corresponding embeddings and let $i_{(X, A)}^{(k)}:(X, A) \rightarrow C^{2}\left(g_{k}\right)$ and $j_{(Z, C)}^{(k)}:(Z, C) \rightarrow C^{2}\left(g_{k}\right)$ as well as $j_{(Z, C)}:(Z, C) \rightarrow C^{2}(\phi)$ be the canonical embeddings as above (cf. Theorem 3.2). Then

$$
\begin{aligned}
j_{(Z, C)} \circ g_{0} & =\alpha_{0} \circ j_{(Z, C)}^{(0)} \circ g_{0} \simeq \alpha_{0} \circ i_{(X, A)}^{(0)} \simeq \alpha_{1} \circ i_{(X, A)}^{(1)} \\
& \simeq \alpha_{1} \circ j_{(Z, C)}^{(1)} \circ g_{1} \simeq j_{(Z, C)} \circ g_{1} .
\end{aligned}
$$

Since $j_{(Z, C)}$ is a cylinder base embedding, we are done.

The a fortiori-part of the theorem holds because strong shape equivalences of pairs are precisely the morphisms which are taken to isomorphisms by $Q S$. 


\section{References}

[1] F.W. Bauer, A shape theory with singular homology, Pacific J. Math. 64 (1976), 25-65.

[2] F.W. Bauer, Under what conditions are shape homology $\underline{E}_{*}$ and Steenrod homology ${ }^{s} \underline{E}_{*}$ isomorphic?, in: Shape theory and geometric topology (Proceedings Dubrovnik 1981), 186-214, Lecture Notes in Math. 870, Springer-Verlag, New York, 1981.

[3] F.W. Bauer, Extensions of generalized homology theories, Pacific J. Math. 128 (1987), 25-61.

[4] L.G. Brown, R.G. Douglas, and P.A. Fillmore, Extensions of $C^{*}$-algebras and K-homology, Ann. of Math. (2) 105 (1977), no. 2, 265-324.

[5] D.A. Edwards and H.M. Hastings, Čech and Steenrod homotopy theories with applications to geometric topology, Lecture Notes in Math. 542, SpringerVerlag, New York, 1976.

[6] B. Günther, Properties of normal embeddings concerning strong shape theory, II, Tsukuba J. Math. 16 (1992), 429-438.

[7] S. Mardešić, Strong shape and homology, Springer-Verlag, New York, 2000.

[8] S. Mardešić, Strong expansions and strong shape for pairs of spaces, Rad Hrvatske Akad. Znan. Umjet. Matem. Znan. 456 (1991), 159-172.

[9] J. Milnor, On the Steenrod homology theory, mimeographed notes, Berkeley, 1960; also in: Novikov conjectures, index theorems and rigidity, Vol. 1 (S.C. Ferry, A. Ranicki, J. Rosenberg, eds.), London Math. Soc. Lecture Note Series 226, Cambridge University Press, Cambridge, 1995, 79-96.

[10] P. Mrozik, Mapping cylinders of approaching maps and strong shape, J. London Math. Soc. (2) 41 (1990), no. 1, 159-174.

[11] J.B. Quigley, An exact sequence from the $n$th to $(n-1)$-st fundamental group, Fund. Math. 91 (1973), no. 3, 195-210.

[12] A.D. Wallace, The map excision theorem, Duke Math. J. 19 (1952), 177-182.

Peter Mrozik pmrozik@gmx.de

Berliner Str. 11, 61194 Niddatal, Germany 